\title{
A Nonstandard Generalization of Envelopes
}

Tahir H. Ismail

tahir_hsis@uomosul.edu.iq

College of computers Sciences

and Mathematics

University of Mosul

Received on: 27/6/2007

\section{Ibrahim O. Hamad}

ibrahim.hamad@su.edu.krd

College of Sciences

Salahaddin University

Accepted on: 4/11/2007

\section{ABSTRACT}

The generalized envelopes are studied by a given nonstandard definition of envelope of a family of lines defined in a projective homogenous coordinates PHC by: $\mathrm{u}(\mathrm{t}) x+\mathrm{v}(\mathrm{t}) y+\mathrm{w}(\mathrm{t}) z=0$. The new nonstandard concepts of envelope are applied to conic sections. Our goal in this paper is hat for a given conic section curve $f(\mathrm{x}, \mathrm{y})=0$, we search for the family of lines in which $f$ is its envelope.

Keywords: infinitesimals, monad, envelope.

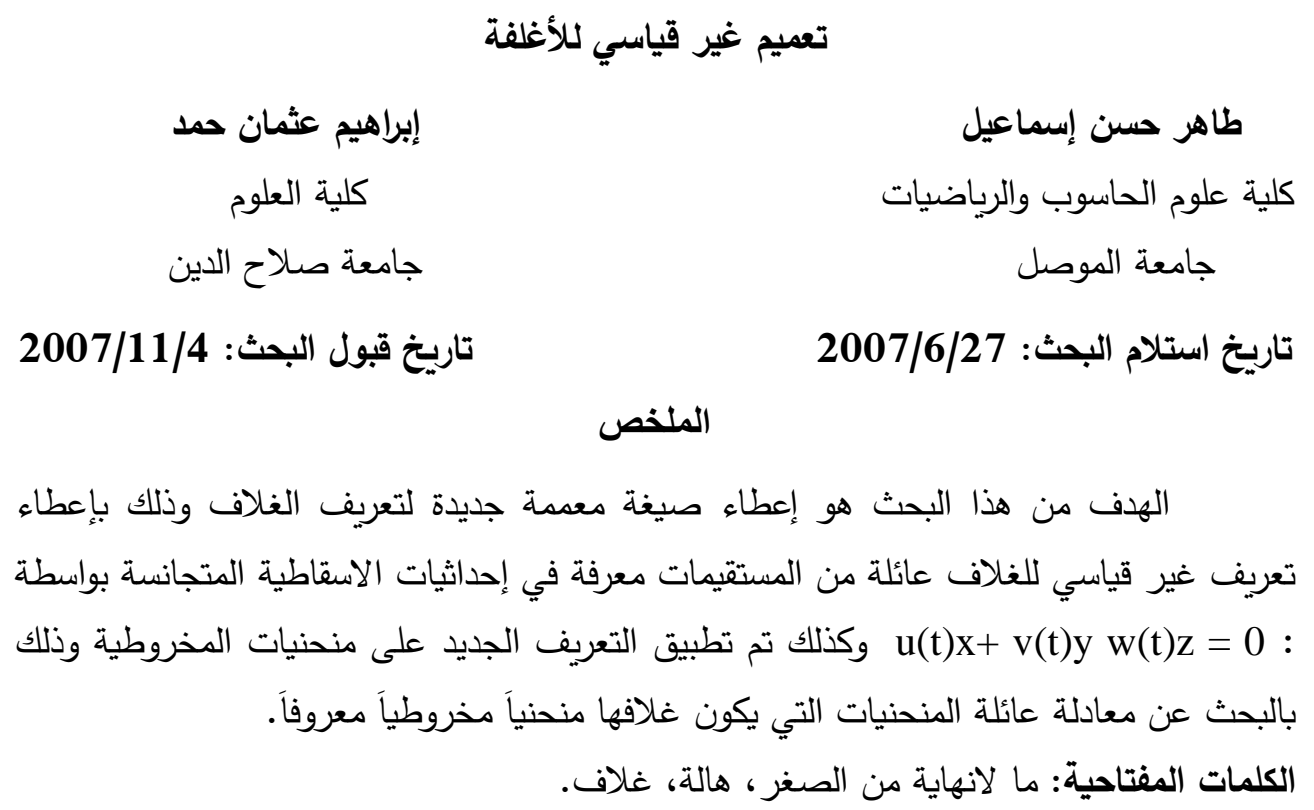

\section{1- Introduction:}

The following definitions and notations are needed throughout this paper. 
Every concept concerning sets or elements defined in the classical mathematics is called standard [7].

Any set or formula which does not involve new predicates "standard, infinitesimals, limited, unlimited...etc" is called internal, otherwise it is called external. [7]

A real number $\boldsymbol{x}$ is called unlimited if and only if $|\boldsymbol{x}|>\boldsymbol{r}$ for all positive standard real numbers; otherwise it is called limited [4].

A real number $\boldsymbol{x}$ is called infinitesimal if and only if $|\boldsymbol{x}|<\boldsymbol{r}$ for all positive standard real numbers $\boldsymbol{r}$ [6], [4].

Two real numbers $x$ and $y$ are said to be infinitely close if and only if $\boldsymbol{x}-\boldsymbol{y}$ is infinitesimal and denoted by $\boldsymbol{x} \cong \boldsymbol{y}[8]$.

If $\boldsymbol{x}$ is a limited number in $\mathbf{R}$, then it is infinitely close to a unique standard real number, this unique number is called the standard part of $\boldsymbol{x}$ or shadow of $\boldsymbol{x}$ denoted by $\operatorname{st}(x)$ or ${ }^{0} \boldsymbol{x}$ [6], [8].

\section{Theorem 1.1 :( Extension Principle) [3]}

Let $\square \mathbf{X} \square$ and $\mathbf{Y}$ be two standard sets, ${ }^{s} \mathbf{X}$ and ${ }^{s} \mathbf{Y}$ be the subsets constitute of the standard elements of $\square \mathbf{X} \square \square \square$ and $\mathbf{Y}$, respectively. If we can associate with every $\boldsymbol{x} \in{ }^{s} \mathbf{X}$ a unique $\boldsymbol{y}=\boldsymbol{f}(\boldsymbol{x}) \in{ }^{s} \mathbf{Y}$ then there exists a unique standard $\boldsymbol{y}^{*} \in \mathbf{Y}$ such that $\forall^{s t} \boldsymbol{x} \in \chi, \boldsymbol{y}^{*}=\boldsymbol{f}(\boldsymbol{x})$

Let $\alpha$ and $\beta$ be any two infinitesimal numbers and $r \neq 0$ is a limited real number, then:
1. $\alpha \cdot r$ is an infinitesimal.
2. $\square \alpha \cdot \beta$ is an infinitesimal.

3. $\alpha+r$ is limited.

4. $\alpha+\beta$ is an infinitesimal (in general the sum of any arbitrary finite number of infinitesimal numbers is infinitesimal) [6].

The projective plane over $\mathbf{R}$, denoted by $\mathbf{P}_{\mathbf{R}}^{\mathbf{2}}$ is the set $\mathbf{P}_{\mathbf{R}}^{\mathbf{2}}=\mathbf{R}^{\mathbf{2}} \cup\{$ one point at $\infty$ for each equivalence classes of parallel lines $\}$,we denoted it by $(\boldsymbol{P H P})[2]$.

The projective homogeneous coordinates of a point $\boldsymbol{p}(\boldsymbol{x}, \boldsymbol{y}) \in \mathbf{R}^{\mathbf{2}}$ are $[\boldsymbol{x} \alpha, y \boldsymbol{\alpha}, \boldsymbol{\alpha}]$, where $\boldsymbol{\alpha}$ is any nonzero number, we denoted it by $(\boldsymbol{P H C}$, in this sense the projective homogeneous coordinates of any point is not unique [2].

A curve $\boldsymbol{v}$ is called envelope of a family of curves $\gamma_{\alpha}$ depending on a parameter $\boldsymbol{\alpha}$, if at each of its points, it is tangent to at least one curve of the family, and if each of its segments is tangent to an infinite set of these curves[2]. 
By a parameterized differentiable curve, we mean a differentiable map $\gamma: \mathrm{I} \rightarrow \mathbf{R}^{\mathbf{3}}$ of an open interval $\mathbb{I}=(\boldsymbol{a}, \boldsymbol{b})$ of the real line $\mathbf{R}$ in to $\mathbf{R}^{\mathbf{3}}$ such that: $\gamma(t)=(x(t), y(t), z(t))=x(t) e_{1}+y(t) e_{2}+z(t) e_{3}$, and $x, y$, and $z$ are differentiable at $\mathrm{t}$; it is also called spherical curve[2].

\section{An Envelope of a Family of Lines in a Plane}

We consider $\mathbf{R}^{\mathbf{2}}$ as a subset of $\boldsymbol{P H P}$, let $\left\{\boldsymbol{L}_{t}\right\}$ be a family of lines in $\boldsymbol{P H C}$ space defined by:

$$
u(t) \mathbf{X}+v(t) \mathbf{Y}+w(t) \mathbf{Z}=\mathbf{0}
$$

and suppose that the ordered pairs $(\boldsymbol{u}(t), v(t)),(\boldsymbol{u}(t), w(t)),(v(t), w(t))$, where $u$, $v, w$ are standard functions defined on an interval sub set of $\mathbf{R}$.

The purpose is to associate a standard curve which is coincident with the envelope to the family $\left\{\boldsymbol{L}_{t}\right\}$.

Suppose that $\boldsymbol{t}$ ranges over the interval $\boldsymbol{E} \subset \mathbf{R}$ so that for every $\boldsymbol{t} \in \boldsymbol{E}$, there exists $\boldsymbol{\alpha}>\boldsymbol{O}$ such that $\forall \boldsymbol{s} \in[t-\alpha, t+\alpha], \boldsymbol{L}_{t} \neq \boldsymbol{L}_{s}$

This is equivalent to $\boldsymbol{L}_{t} \neq \boldsymbol{L}_{t+\varepsilon} \forall \boldsymbol{t} \in \boldsymbol{E}$, where $\boldsymbol{\varepsilon}$ is an infinitesimal real number.

Also, at each standard $t$, we can associate two lines $\boldsymbol{L}_{t}$ and $\boldsymbol{L}_{t+\varepsilon}$ such that $\boldsymbol{L}_{\boldsymbol{t}} \neq \boldsymbol{L}_{t+\varepsilon}$, where $\boldsymbol{L}_{\boldsymbol{t}}$ and $\boldsymbol{L}_{t+\varepsilon}$ are taken in $\boldsymbol{P H P}$.

Let $\gamma(\boldsymbol{t})$ be the envelope curve of the family $\left\{\boldsymbol{L}_{t}\right\}$. By using the principle of extension we have:

There exists a unique standard application $\alpha: E \rightarrow P_{R}^{2}$ such that $\gamma(\boldsymbol{t}) \equiv \alpha(\mathrm{t}) \forall^{s t} \boldsymbol{t} \in \boldsymbol{E}$.

Now, let the families $\left\{\boldsymbol{L}_{t}\right\}$ and $\left\{\boldsymbol{L}_{t+\varepsilon}\right\}$ be given as follows:

$$
\begin{aligned}
& L_{t}: u(t) X+v(t) Y+w(t) Z=0 \\
& L_{t+\varepsilon}: u(t+\varepsilon) X+v(t+\square \varepsilon) Y+w(t+\square \varepsilon) Z=0 .
\end{aligned}
$$

Then the intersection point of $\left\{\boldsymbol{L}_{t}\right\}$ and $\left\{\boldsymbol{L}_{\boldsymbol{t}+\varepsilon}\right\}$ in $\boldsymbol{P H C}$ is given by:

$$
\begin{aligned}
& X_{\varepsilon}(t)=v(t+\varepsilon) w(t)-v(t) w(t+\varepsilon) \\
& Y_{\varepsilon}(t)=w(t+\varepsilon) u(t)-w(t) u(t+\varepsilon) \\
& Z_{\varepsilon}(t)=u(t+\varepsilon) v(t)-u(t) v(t+\varepsilon)
\end{aligned}
$$

Suppose that the functions $\boldsymbol{u}, \boldsymbol{v}$, and $\boldsymbol{w}$ are differentiable functions each of order at least $\boldsymbol{n}$, then by expanding each of $\boldsymbol{u}(\boldsymbol{t}+\varepsilon), \boldsymbol{v}(\boldsymbol{t}+\varepsilon)$, and $\boldsymbol{w}(\boldsymbol{t}+\varepsilon)$ using Taylor development, we get

$X \varepsilon(t)=v(t+\varepsilon) w(t)-v(t) w(t+\varepsilon)$ 


$$
\begin{aligned}
& =\left(v^{\prime}(t) w(t)-w^{\prime}(t) v(t)\right) \varepsilon+\cdots+\left(v^{(\mathrm{n})}(t) w(t)-w^{(\mathrm{n})}(t) v(t)\right) \frac{\varepsilon^{n}}{n !}+\delta_{1} \varepsilon^{n} \\
\boldsymbol{Y}_{\varepsilon}(t) & =w(t+\varepsilon) u(t)-w(t) u(t+\varepsilon) \\
& =\left(w^{\prime}(t) u(t)-u^{\prime}(t) w(t)\right) \varepsilon+\cdots+\left(w^{(\mathrm{n})}(t) u(t)-u^{(\mathrm{n})}(t) w(t)\right) \frac{\varepsilon^{n}}{n !}+\delta_{2} \varepsilon^{n} \\
Z_{\varepsilon}(t) & =u(t+\varepsilon) v(t)-u(t) v(t+\varepsilon) \\
& =\left(u^{\prime}(t) v(t)-v^{\prime}(t) u(t)\right) \varepsilon+\cdots+\left(u^{(\mathrm{n})}(t) v(t)-v^{(\mathrm{n})}(t) u(t)\right) \frac{\varepsilon^{n}}{n !}+\delta_{3} \varepsilon^{n},
\end{aligned}
$$

where $\boldsymbol{\delta}_{1}, \boldsymbol{\delta}_{2}, \boldsymbol{\delta}_{3}$ are infinitesimals. In general, put

$p_{n}(t)=v^{(n)}(t) w(t)-w^{(n)}(t) v(t)$

$r_{n}(t)=w^{(n)}(t) u(t)-u^{(n)}(t) w(t)$

$q_{n}(t)=u^{(n)}(t) v(t)-v^{(n)}(t) u(t)$

The following cases are related to the last assumption

Case1. If $q_{I}(t) \neq \boldsymbol{O}$ and $p_{I}(t)$ and $\boldsymbol{r}_{I}(t)$ are not both zero, then the $\boldsymbol{P H C}$ points of envelope curve $\gamma(t),\left(\boldsymbol{p}_{1}(\boldsymbol{t}), \boldsymbol{r}_{1}(\boldsymbol{t}), \boldsymbol{q}_{1}(\boldsymbol{t})\right)$, are independent on $\varepsilon$, and the triple $\left(p_{1}(t), \boldsymbol{r}_{1}(t), \boldsymbol{q}_{1}(t)\right)$ represents the classical definition of an envelope curve.

\section{Proof:}

Using (2.2), we get:

$$
\begin{aligned}
\mathrm{X} \varepsilon(\mathrm{t}) & =\left(\mathrm{v}^{\prime}(\mathrm{t}) \mathrm{w}(\mathrm{t})-\mathrm{w}^{\prime}(\mathrm{t}) \mathrm{v}(\mathrm{t})\right) \varepsilon+\ldots+(\mathrm{v}(\mathrm{n})(\mathrm{t}) \mathrm{w}(\mathrm{t})-\mathrm{w}(\mathrm{n})(\mathrm{t}) \mathrm{v}(\mathrm{t})) \frac{\varepsilon^{n}}{n !}+\delta_{1} \varepsilon^{n} \\
= & \varepsilon\left(\mathrm{v}^{\prime}(\mathrm{t}) \mathrm{w}(\mathrm{t})-\mathrm{w}^{\prime}(\mathrm{t}) \mathrm{v}(\mathrm{t})\right)+\ldots+(\mathrm{v}(\mathrm{n})(\mathrm{t}) \mathrm{w}(\mathrm{t})-\mathrm{w}(\mathrm{n})(\mathrm{t}) \mathrm{v}(\mathrm{t})) \frac{\varepsilon^{n-1}}{n !}+\delta_{1} \varepsilon^{n-1}
\end{aligned}
$$

Taking the shadow of the of the last equation we obtain

$$
{ }^{o} X_{\varepsilon}(t)=\varepsilon\left(v^{\prime}(t) w(t)-w^{\prime}(t) v(t)\right)
$$

In the same way, we have

$$
{ }^{o} Y_{\varepsilon}(t)=\varepsilon\left(w^{\prime}(t) u(t)-u^{\prime}(t) w(t)\right),
$$

and

$$
{ }^{o} Z \varepsilon(t)=\varepsilon\left(u^{\prime}(t) v(t)-v^{\prime}(t) u(t)\right)
$$

Therefore,

$$
\begin{aligned}
& \left(X_{\varepsilon}(t), Y_{\varepsilon}(t), Z_{\varepsilon}(t)\right)>\left({ }^{o} X_{\varepsilon}(t),{ }^{o} Y \varepsilon(t),{ }^{o} Z_{\varepsilon}(t)\right) \\
& =\left(\varepsilon\left(v^{\prime}(t) w(t)-w^{\prime}(t) v(t)\right), \varepsilon\left(w^{\prime}(t) u(t)-u^{\prime}(t) w(t)\right), \varepsilon\left(u^{\prime}(t) v(t)-v^{\prime}(t) u(t)\right)\right)
\end{aligned}
$$

Now, using the properties of the $\boldsymbol{P H C}$, we deduce that any point of the form $(\lambda \boldsymbol{a}, \lambda \boldsymbol{b}, \boldsymbol{\lambda} \boldsymbol{c})$ is equivalent with the point $(\boldsymbol{a}, \boldsymbol{b}, \boldsymbol{c})$ for any parameter $\boldsymbol{\lambda}$. 
Therefore, the $\boldsymbol{P H C}$ of $\gamma(\boldsymbol{t})$ is $\left(\boldsymbol{X} \varepsilon(\boldsymbol{t}), \boldsymbol{Y} \varepsilon(\boldsymbol{t}), \boldsymbol{Z}_{\varepsilon}(\boldsymbol{t})\right)$, and it is equal to

$$
\begin{aligned}
& \left(v^{\prime}(t) w(t)-w^{\prime}(t) v(t), w^{\prime}(t) u(t)-u^{\prime}(t) w(t), u^{\prime}(t) v(t)-v^{\prime}(t) u(t)\right) \\
& =\left(p_{I}(t), r_{I}(t), q_{I}(t)\right)
\end{aligned}
$$

That is, $\left(\boldsymbol{p}_{I}(\boldsymbol{t}), \boldsymbol{r}_{I}(\boldsymbol{t}), \boldsymbol{q}_{I}(\boldsymbol{t})\right)$ represents the classical definition of an envelope curve which does not depend on $\varepsilon$.

Moreover, the Cartesian coordinates of points of $\gamma$ are given by

$$
\begin{aligned}
(x(t), y(t)) & =\left(\frac{X_{\varepsilon}(t)}{Z_{\varepsilon}(t)}, \frac{Y_{\varepsilon}(t)}{Z_{\varepsilon}(t)}\right) \\
& =\left(\frac{v^{\prime}(t) w(t)-w^{\prime}(t) v(t)}{u^{\prime}(t) v(t)-v^{\prime}(t) u(t)}, \frac{w^{\prime}(t) u(t)-u^{\prime}(t) w(t)}{u^{\prime}(t) v(t)-v^{\prime}(t) u(t)}\right)
\end{aligned}
$$

This is the classical form of the envelope curve of the family of straight lines.

Case 2. If $q_{1}(t)=\boldsymbol{O}, p_{1}(t)$, and $\boldsymbol{r}_{1}(\boldsymbol{t})$ are not both zeroes, then the $\boldsymbol{P H C}$ points of the envelope curve $\gamma(\boldsymbol{t})$ are infinitely large, and the corresponding tangents $\left\{\boldsymbol{L}_{t}\right\}$ are asymptotes of $\gamma(\boldsymbol{t})$.

Case3. If $p_{1}(t)=r_{1}(t)=q_{1}(t)=0$ and $p_{2}(t) \neq 0, r_{2}(t) \neq 0=q_{2}(t) \neq 0$ then, the PHC points of the envelope curve $\gamma(\boldsymbol{t})$ are $\left(\boldsymbol{p}_{2}(\boldsymbol{t}), \boldsymbol{r}_{2}(\boldsymbol{t}), \boldsymbol{q}_{2}(\boldsymbol{t})\right)$, and $\left(\boldsymbol{p}_{1}(t), \boldsymbol{r}_{1}(t), \boldsymbol{q}_{1}(t)\right)$ is an inflection point of the envelope curve $\gamma(\boldsymbol{t})$.

Case4. If $\boldsymbol{p}_{\boldsymbol{k}}(\boldsymbol{t})=\boldsymbol{r}_{\boldsymbol{k}}(\mathrm{t})=\boldsymbol{q}_{\boldsymbol{k}}(\boldsymbol{t})=\mathbf{0}$ for $\boldsymbol{1} \leq \boldsymbol{k}<\boldsymbol{n}$ (n standard) and $\boldsymbol{p}_{\boldsymbol{n}}(\boldsymbol{t}), \boldsymbol{r}_{\boldsymbol{n}}(\boldsymbol{t})$, $\boldsymbol{q}_{n}(\boldsymbol{t})$ are not all zeros, then the $\boldsymbol{P H C}$ points of $\gamma(\boldsymbol{t})$ are of the form $\left(\boldsymbol{p}_{n}(t)\right.$, $\left.\boldsymbol{r}_{n}(\boldsymbol{t}), \boldsymbol{q}_{n}(\boldsymbol{t})\right)$ which does not depend on $\varepsilon$. Thus, we get the generalized nonclassical form of the envelope curve $\gamma(\boldsymbol{t})$ as follows:

$$
\begin{aligned}
(x(t), y(t)) & =\left(\frac{X_{\varepsilon}(t)}{Z_{\varepsilon}(t)}, \frac{Y_{\varepsilon}(t)}{Z_{\varepsilon}(t)}\right) \\
& =\left(\frac{v^{(n)}(t) w(t)-w^{(n)}(t) v(t)}{u^{(n)}(t) v(t)-v^{(n)}(t) u(t)}, \frac{w^{(n)}(t) u(t)-u^{(n)}(t) w(t)}{u^{(n)}(t) v(t)-v^{(n)}(t) u(t)}\right)
\end{aligned}
$$

Case5. If $p_{k}(t)=r_{k}(t)=q_{k}(t)=0$ for any value of $\boldsymbol{k}$, then we can not say any thing about the generalization of the envelope curve. In the following sections, by $p(t), r(t)$ and $q(t)$ we mean $p_{I}(t), r_{I}(t)$ and $q_{I}(t)$ respectively. 


\section{Applications to Conic Sections}

We restrict our study on a family of straight lines only, other studies on envelops and singularity of envelops, for example can be found in [1]. Our goal is that for a given conic section curve $\boldsymbol{f}(\boldsymbol{x}, \boldsymbol{y})=\mathbf{0}$, we search for a family of lines in which $f$ is its envelope.

\section{Lemma 3.1}

Consider a standard family of lines $\left\{\boldsymbol{L}_{t}\right\}$ defined by

$u(t) X+v(t) Y+w(t) Z=0$

where $\boldsymbol{u}, \boldsymbol{v}$, and $\boldsymbol{w}$ are standard real polynomials of at most second degree, then the equation of $\left\{\boldsymbol{L}_{t}\right\}$ can be written as follows:

$A(X, Y, Z) t^{2}+B(X, Y, Z) t+C(X, Y, Z)=0$,

in which $\boldsymbol{A}, \boldsymbol{B}$, and $\boldsymbol{C}$ are linear equations of the variables $\boldsymbol{X}$ and $\boldsymbol{Y}$ and $\boldsymbol{Z}$ belonging to $\boldsymbol{P H P}$. And conversely every equation of the form (3.1.2) represents a family of lines of the form (3.1.1)

Proof:

Obvious

\section{Theorem 3.2}

If $\left\{\boldsymbol{L}_{t}\right\}$ is a family of lines defined by:

$u(t) x+v(t) y+w(t)=0$,

where $\boldsymbol{u}, \boldsymbol{v}$, and $\boldsymbol{w}$ are standard real polynomials of at most second degree, then the envelope of $\left\{\boldsymbol{L}_{t}\right\}$ is a cone of the form:

$B^{2}(x, y)-4 A(x, y) C(x, y)=0$,

in which $\boldsymbol{A}, \boldsymbol{B}$, and $\boldsymbol{C}$ are linear equations of the variables $\boldsymbol{x}$ and $\boldsymbol{y}$ belonging to $\mathbb{R}[x, y]$.

Moreover Equation (3.2.1) represents a general form of a second degree equation of two variables $\boldsymbol{x}$ and $\boldsymbol{y}$ and conversely.

Proof:

Consider the families of lines $\left\{\boldsymbol{L}_{t}\right\}$ and $\left\{\boldsymbol{L}_{\boldsymbol{t}+\varepsilon}\right\}$

By using Lemma 3.1, we get that:

$$
\begin{aligned}
& L_{t}: A(x, y) t^{2}+B(x, y) t+C(x, y)=0 \\
& L_{t+\varepsilon}: A(x, y)(t+\varepsilon)^{2}+B(x, y)(t+\varepsilon)+C(x, y)=0
\end{aligned}
$$

Then solving $\boldsymbol{L}_{\boldsymbol{t}}$ and $\boldsymbol{L}_{\boldsymbol{t}+\varepsilon}$ as an instantaneous system to omit $\boldsymbol{t}^{2}$ we get:

$2 \varepsilon \boldsymbol{A}(\boldsymbol{x}, \boldsymbol{y}) \boldsymbol{t}+\boldsymbol{A}(\boldsymbol{x}, \boldsymbol{y}) \varepsilon^{2}+\boldsymbol{B}(\boldsymbol{x}, \boldsymbol{y}) \varepsilon=\mathbf{0}$. 
Therefore,

$2 A(x, y) t+A(x, y) \varepsilon+B(x, y)=0$

Taking the shadow of (3.2.2), we get $t=\frac{-\boldsymbol{B}(\boldsymbol{x}, \boldsymbol{y})}{2 \boldsymbol{Q A}(\boldsymbol{x}, \boldsymbol{y})}$ and then putting it in $\boldsymbol{L}_{\boldsymbol{t}}$ we obtain the required result.

For the second part, since $\boldsymbol{A}, \boldsymbol{B}$, and $\boldsymbol{C}$ are linear equations of the variables $\boldsymbol{x}$ and $\boldsymbol{y}$ belonging to $\mathbb{R}[\boldsymbol{x}, \boldsymbol{y}]$, so Putting

$A(x, y)=a_{1} x+a_{2} y+a_{3}$

$B(x, y)=b_{1} x+b_{2} y+b_{3}$

$C(x, y)=c_{1} x+c_{2} y+c_{3}$,

in Equation (3.2.1), we get the following equation

$B^{2}(x, y)-4 A(x, y) C(x, y)=a x^{2}+b x y+c y^{2}+d x+e y+f=0$,

where

$a=\left(b_{1}^{2}-4 a_{1} c_{1}\right)$

$b=2\left(b_{1} b_{2}-2\left(a_{1} c_{2}+a_{2} c_{1}\right)\right)$

$c=\left(b_{2}^{2}-4 a_{2} c_{2}\right)$

$d=2\left(b_{1} b_{3}-2\left(a_{1} c_{3}+a_{3} c_{1}\right)\right)$

$e=2\left(b_{2} b_{3}-2\left(a_{2} c_{3}+a_{3} c_{2}\right)\right)$

$f=\left(b_{3}^{2}-4 a_{3} c_{3}\right)$

This is a general form of second degree equation in two variables $\boldsymbol{x}$ and $y$.

Conversely, assuming that we have a second degree equation of two variables $\boldsymbol{x}$ and $\boldsymbol{y}$ such as:

$a x^{2}+b x y+c y^{2}+d x+e y+f=0$

By a suitable changing of coordinate's axis; if $\boldsymbol{b} \neq \boldsymbol{0}$ then a rotation of axis through the angle $\alpha$ determined by the equation $\cot 2 \alpha=\frac{A-C}{B}$ will transform Equation (3.2.3) to the following equation

$a^{*} x^{2}+b^{*} y^{2}+c^{*} x+d^{*} y+e^{*}=0$

Completing the square for each uncompleted square related to the variables $\boldsymbol{x}$ and $\boldsymbol{y}$ in Equation (3.2.4) and simplifying the result, we get: 


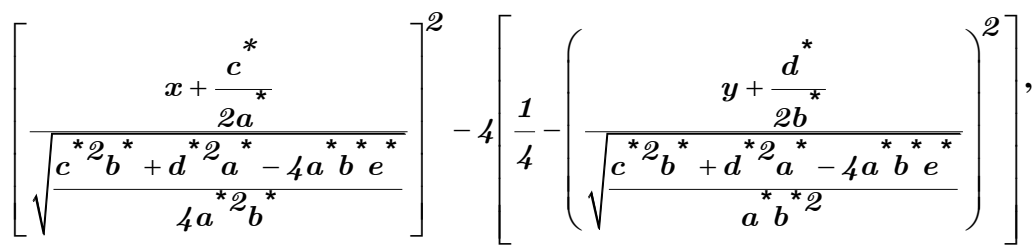

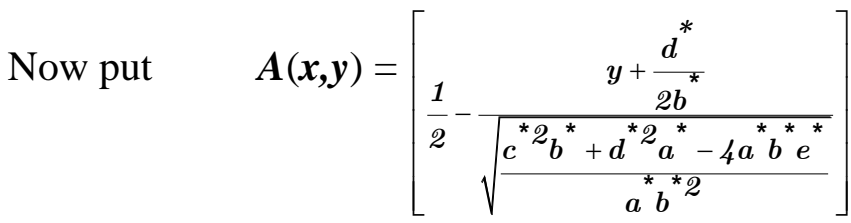

$\boldsymbol{B}(\boldsymbol{x}, \boldsymbol{y})=\left[\frac{x+\frac{c^{*}}{2 a^{*}}}{\sqrt{\frac{c^{{ }^{*}{ }^{*} b^{*}+d^{*}{ }^{*} a^{*}-4 a^{*} b^{*} e^{*}}}{4 a^{{ }^{*}} 2^{*}}}}\right]$

$C(x, y)=\left[\frac{1}{2}+\frac{y+\frac{d^{*}}{2 b^{*}}}{\sqrt{\frac{c^{*} 2_{b}{ }^{*}+d^{*} \mathscr{2}^{*}{ }^{*}-4 a^{*} b^{*} e^{*}}{a^{*} b^{*} \mathscr{2}}}}\right]$

If $a^{*}, b^{*}=0$ or $\frac{c^{*} b^{*}+d^{*} a^{*}-4 a^{*} b^{*} e^{*}}{4 a^{*} b^{*}}<0$ then we obtain undefined or imaginary values which are unacceptable cases in real homogenous projective plane.

Thus we assume that $a^{*}, b^{*} \neq 0$ and $\frac{c^{* 2} b^{*}+d^{*} a^{*}-4 a^{*} b^{*} e^{*}}{4 a^{*} b^{* 2}} \geq 0$.

Hence we get the required result.

\section{Remark 3.3}

If the last conditions of the previous theorem are not valid or $a^{*}=b^{*}$ we deduce that Equation (3.2.4) represents a standard conic section which can be transferred to the form (3.2.1) as it is shown in the following table: 


\begin{tabular}{|c|c|c|c|c|c|}
\hline Conic Section & General Form & Standard Form & $A(x, y)$ & $B(x, y)$ & $C(x, y)$ \\
\hline Circle & $x^{2}+y^{2}+a x+b y+c=0$ & $x^{2}+y^{2}=r^{2}$ & $r / 2-x / 2$ & $y$ & $r / 2+x / 2$ \\
\hline Parabola & $y^{2}+a x+b y+c=0$ & $y^{2}=4 a x$ & $a$ & $y$ & $x$ \\
\hline Ellipse & $a x^{2}+b y^{2}+c x+d y+e=0$ & $x^{2} / a^{2}+y^{2} / b^{2}=1$ & $1 / 2-x / 2 a$ & $y / b$ & $1 / 2+x / 2 a$ \\
\hline Hyperbolic & $a x^{2}+b y^{2}+c x+d y+e=0$ & $x^{2} / a^{2}-y^{2} / b^{2}=1$ & $x / 2 a-y / 2 b$ & 1 & $x / 2 a+y / 2 b$ \\
\hline
\end{tabular}

\section{Example 3.4}

The circle $\boldsymbol{x}^{2}+\boldsymbol{y}^{2}=\boldsymbol{1}$ is an envelope curve of the family of lines $\left(1-t^{2}\right) x+(2 t) y+\left(t^{2}+1\right)=0$, such as shown in the Figure 3.1

By applying Theorem 3.2 to the equation of the given circle we get

$y^{2}-4(1 / 2-x / 2)(1 / 2+x / 2)=0$

Therefore $\quad(1 / 2-x / 2) t^{2}+y t+1 / 2+x / 2=0$,

which is an equation of a family of lines.

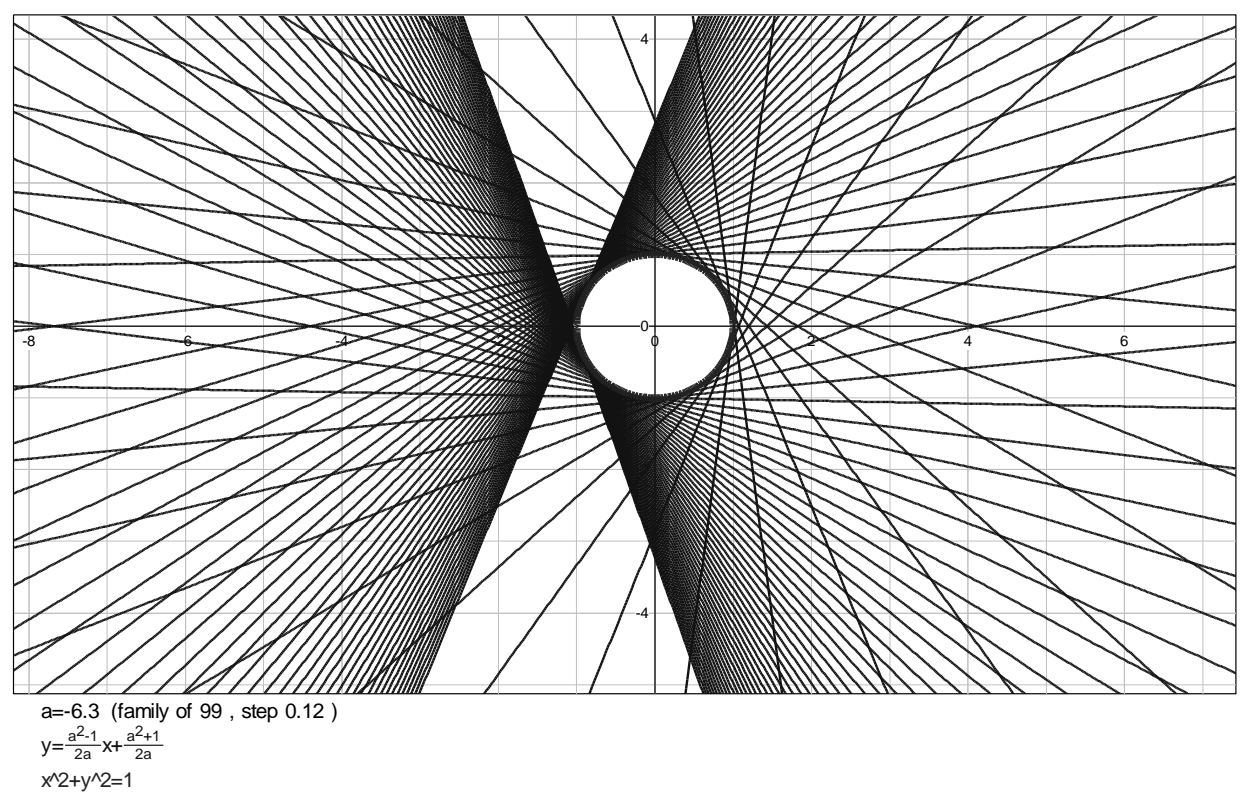


Figure 3.1

Note that we can show that the given circle equation $\boldsymbol{x}^{2}+\boldsymbol{y}^{2}=\boldsymbol{1}$ is an envelope equation of the founded family classically or by nonstandard tools. In the following, we give a nonstandard method for such purpose.

$$
\left.\begin{array}{l}
L_{t}:\left(1-t^{2}\right) x+(2 t) y+\left(t^{2}+1\right)=0 \\
L_{t+\varepsilon}:\left(1-(t+\varepsilon)^{2}\right) x+2(t+\square \varepsilon) y+(t+\square \varepsilon)^{2}+1=0
\end{array}\right\}
$$

Solving equations $\boldsymbol{L}_{\boldsymbol{t}}$ and $\boldsymbol{L}_{\boldsymbol{t}+\varepsilon}$ instantaneously, we get:

$\mathbf{2} \varepsilon \boldsymbol{t} \boldsymbol{x}+\varepsilon^{2} \boldsymbol{x}-\mathbf{2} \varepsilon \boldsymbol{y}-\mathbf{2} \varepsilon \boldsymbol{t}-\varepsilon^{2}=\mathbf{0}$.

\section{Therefore}

$2 t x+\square \varepsilon x-2 y-2 t-\square \varepsilon=0$.

Taking the shadow, we get

$2 t x-2 y-2 t=0$

Now, remove the variable $t$ form Equations (3.4.1) and (3.4.2), we get the required result.

\section{Example 3.5}

Consider the curve $\boldsymbol{x}+\boldsymbol{y}^{2}-\mathbf{1}=\mathbf{0}$

By applying Theorem 3.2 to the given equation, we get

$y^{2}-4(1 / 4)(1-x)=0$,

now use Lemma 3.1 we get

$1 / 4 t^{2}+y t+1-x=0$

which is a family of lines whose envelope is the given equation, such as shown in the Figure 3.2

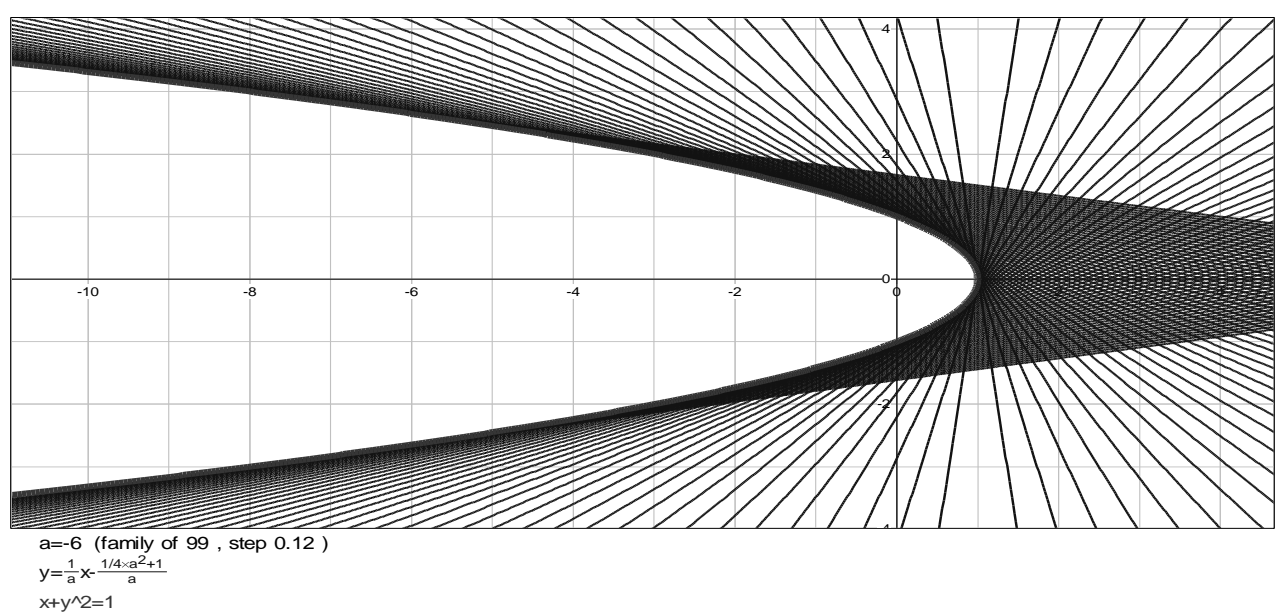

Figure 3.2 
Remark: The graphs in Figs 3.1 and 3.2 are plotted with specific softwares: Omnigraph V3.1b-2005. ,Function Grapher V2.8-2006.

\section{$\underline{\text { REFERENCE }}$}

[1] Capitanio, G. (2005); Cusp Singularities of Plane Envelopes,[arXiv math. DG/0511511 v1,20].

[2] Carmo, M. (1976); Differential Geometry of Curves and Surfaces, Prentice-Hall, INC, New York.

[3] Diener, F. (1983); Course d'Analyse Nonstandard, Office des Pub. Universities, Alger.

[4] Diener, F.\& Diener M. (1996); Nonstandard Analysis in Practice, Springer-Verlag, Berlin,HeildeBerg.

[5] Keisler, H. J.(2005); Elementary Calculus-2ed-An Infinitesimal Approach, Creative Commons, 559 Nathan Abbott, Stanford, California, 93405, USA.

[6] Lutz R. and Goze M. (1982); Nonstandard Analysis-A Practical Guide with Applications, Lecture. Notices in math (881) Springer, Berlin.

[7] Nelson, E. (1977); Internal set Theory-A New Approach to Nonstandard Analysis, Bull. Amer. Math. Soc, Vol.83, No.6, pp.1165-1198.

[8] Rosinger, E. E(2004); Short Introduction to Nonstandard Analysis, arXiv: math. GM/ 0407178 v1 10 Jul. 